\section{A Serrate-expressing signaling center controls Drosophila hematopoiesis}

\author{
Tim Lebestky, 1,3,4 Seung-Hye Jung, ${ }^{1,4}$ and \\ Utpal Banerjee ${ }^{1,2,5}$
}

\begin{abstract}
${ }^{1}$ Molecular Biology Institute, ${ }^{2}$ Department of Molecular, Cell, and Developmental Biology, Biological Chemistry, and Human Genetics, University of California Los Angeles, Los Angeles, California 90095, USA
\end{abstract}

The differentiation of Drosophila blood cells relies on a functional hierarchy between the GATA protein, Serpent (Srp), and multiple lineage-specific transcription factors, such as the AML1-like protein, Lozenge (Lz). Two major branches of Drosophila hematopoiesis give rise to plasmatocytes/macrophages and crystal cells. Serrate signaling through the Notch pathway is critical in the regulation of $\mathrm{Lz}$ expression and the specification of crystal cell precursors, thus providing a key distinction between the two lineages. The expression of Serrate marks a discrete cluster of cells in the lymph gland, a signaling center, with functional similarities to stromal signaling in mammalian hematopoiesis.

Received November 8, 2002; revised version accepted December 9, 2002.

Runx1, also known as Acute Myeloid Leukemia 1 (AML1), is essential for the development of blood cells arising from all lineages of definitive hematopoiesis in mice (Okuda et al. 1996; Wang et al. 1996). In humans, AML1 is the most frequent target for translocations resulting in acute myeloid leukemias (Werner et al. 1999). The family of GATA transcription factors is also used reiteratively in multiple stages of blood development (Orkin 1998), and a cofactor for the GATA proteins, FOG1, is required for erythropoiesis and differentiation of megakaryocytes (Tsang et al. 1998). In studying the regulation of such transcription factors, in vitro differentiation assays have suggested that multiple signaling pathways are coordinately involved in hematopoiesis (Van Den Berg et al. 1998; Yoshida et al. 1998; Milner and Bigas 1999). Unfortunately, there is a paucity of in vivo loss-of-function data involving signaling pathways that control hematopoiesis in mammals due to difficulties such as pleiotropy, redundancy, and early lethality.

There are significant differences with regard to the variety and function of Drosophila hemocytes relative to mammalian blood cells, however, several molecular as-

[Keywords: Serrate; Notch; $\mathrm{Su}(\mathrm{H})$; crystal cell; Drosophila; hematopoiesis]

${ }^{3}$ Present address: Department of Biology 216-76, California Institute of Technology, Pasadena, California 91125, USA.

${ }^{4}$ These authors contributed equally to this work.

${ }^{5}$ Corresponding author.

E-MAIL banerjee@mbi.ucla.edu; FAX (310) 206-9062.

Article and publication are at http://www.genesdev.org/cgi/doi/10.1101/ gad.1052803. pects of early hematopoiesis and immunity have been evolutionarily conserved (Rehorn et al. 1996; Dearolf 1998; Hoffmann et al. 1999; Lebestky et al. 2000). Two major classes of Drosophila hemocytes are plasmatocytes, which can function as macrophages that phagocytose invading pathogens and debris from apoptotic cells (Tepass et al. 1994), and crystal cells that are involved in the melanization of pathogens (Rizki et al. 1980). serpent $(\operatorname{srp})$, a Drosophila GATA homolog, is expressed in all hemocyte precursors and is required for the development of both classes of hemocytes (Rehorn et al. 1996; Lebestky et al. 2000). lozenge (lz) encodes an AML1/Runt domain family transcription factor (Daga et al. 1996) that is expressed in the crystal cell precursors and is required for the specification of this lineage (Rizki and Rizki 1981; Lebestky et al. 2000). glial cells missing ( $\mathrm{gcm})$, a novel transcription factor expressed exclusively in plasmatocytes in the embryo (Bernardoni et al. 1997), is required for their development. Its possible role in larval hematopoiesis is less clear. Srp is essential for the expression of both $\mathrm{lz}$ and $\mathrm{gcm}$ (Bernardoni et al. 1997; Lebestky et al. 2000), creating a hierarchy of transcription factors controlling the two major branches of hematopoiesis (Lebestky et al. 2000). u-shaped (ush), a FOG homolog, also functions in Drosophila hematopoiesis (Fossett et al. 2001). The molecular similarities between Srp/ Ush/Lz and GATA/FOG/AML1 suggest that aspects of molecular mechanisms of blood development are shared between mammals and Drosophila. Previous studies in Drosophila have shown a role for JAK/STAT and Toll pathways in the proliferation of hemocytes and immunity (Dearolf 1998; Mathey-Prevot and Perrimon 1998; Qiu et al. 1998). However, no signaling pathway was known to specify a commitment that distinguishes between lineages.

In this study, we show that localized Notch signaling causes an important early distinction between the crystal cell and plasmatocyte lineages. The Notch pathway controls binary cell fate decisions among undetermined precursor cells in a multitude of developmental systems (Artavanis-Tsakonas et al. 1999). We also find that a signaling center expressing the ligand Serrate is important for differentiation and proliferation of larval hemocytes in Drosophila.

\section{Results and Discussion}

Notch and $\mathrm{Su}(\mathrm{H})$ are required for hemocyte proliferation and development

Morphological and molecular differentiation of hemocytes can be monitored in the larval lymph gland (Shrestha and Gateff 1982). Srp is expressed in all hemocyte progenitors, whereas the expression of $\mathrm{Lz}$ is largely restricted to a small subset of hemocytes in the first pair (anterior-most) lobes of the lymph gland (Fig. 1A). In the Notch temperature-sensitive allele, $N^{t s 1}, \mathrm{Lz}$ expression is eliminated from the lymph gland at the nonpermissive temperature (Fig. 1B). $\mathrm{Lz}^{+}$cells are also missing in the lymph glands of $\mathrm{Su}(H)^{S F 8} / \mathrm{Su}(H)^{A R 9}$ larvae (Fig. 1C). As $\mathrm{Su}(\mathrm{H})$ is the transcription factor that activates Notch target genes (for review, see Weinmaster 2000), the canonical Notch/Su(H) pathway is important for Lz expression in crystal cell precursors. 

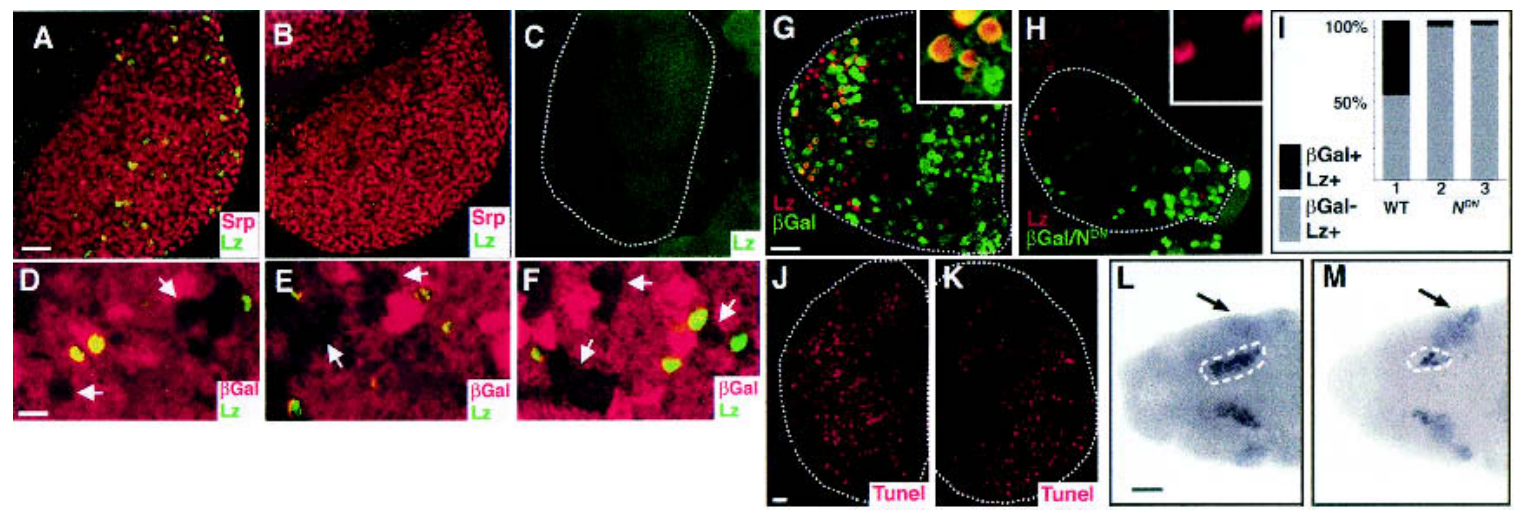

Figure 1. Notch signaling in hematopoiesis. (A) Wild-type first lymph gland lobe from a third instar larva raised at $29^{\circ} \mathrm{C}$ and stained with antibodies against Lz (green) and Serpent (red). Lz is expressed in a distinct subset of Srp ${ }^{+}$hemocytes. $(B) N^{t s 1}$ raised at $29^{\circ} \mathrm{C}$ and stained as in $A$. Lz protein is not expressed, but Srp (red) expression remains unaffected. $(C) S u(H)^{S F 8} / S u(H)^{A R 9}$ lymph gland stained with $\mathrm{Lz}$ antibody. Lz protein is not expressed. $(D-F)$ hsp 70-flp/FRT clones (arrows) of Notch pathway members are marked by the absence of $\beta$ Gal expression (red). Lz expressing cells (green) are excluded from mutant clones of $N^{55 e 11}(D), N^{t s 1}(E)$, and $S u(H)^{\Delta 47}(F)$. $(G)$ hsp $70-f l p ; A y-G a l 4, U A S-\beta G a l /+$. In this control "flp-out" experiment (Ito et al. 1997), $\beta$ Gal-expressing clones were induced in the first larval instar and analyzed in the third instar larval lymph gland. Nuclear $\beta \mathrm{Gal}$ (green) marks cells in which Gal4 is expressed. Many examples of colocalization of Lz and $\beta \mathrm{Gal}$ can be seen (inset). (H) hsp 70-flp; Ay-Gal4, UAS- $\beta$ Gal/UAS-N ${ }^{D N}$. In this genotype, $\beta \mathrm{Gal}^{+}$cells (green) also express $N^{D N}$. $\mathrm{Lz}$ (red) and $\mathrm{N}^{\mathrm{DN}}$ (green) are mutually exclusive (inset), suggesting a requirement for Notch in the development of $\mathrm{Lz}^{+}$cells. Additionally, the lymph gland size is reduced relative to wild-type controls. (I) Cell counts from the Notch clonal analysis described as in $G$ and $H$. For wild-type clones (histogram 1), 20 lymph gland lobes were counted and of 1652 total $\mathrm{Lz}^{+}$cells, 768 were also $\beta \mathrm{Gal}^{+}$. $N^{D N}$ clones were either counted from equivalent number of lymph gland lobes (histogram 2) or equivalent number of $\mathrm{Lz}^{+}$cells (histogram 3). From 20 lymph gland lobes counted (histogram 2), of 153 total $\mathrm{Lz}^{+}$cells, 148 did not express $\beta \mathrm{Gal}$ and, therefore, $N^{D N}$. Similarly, of 1370 total $\mathrm{Lz}^{+}$cells counted from 68 lymph gland lobes (histogram 3 ), only 55 expressed $\beta \mathrm{Gal}$. In either case, $\sim 96 \%$ of the $\mathrm{Lz}^{+}$cells did not express $\beta \mathrm{Gal}$. (J) hsp70-flp; Ay-Gal4, UAS- $\beta$ Gal/+. Experimental conditions are the same as in G. Tunel staining (red) marks apoptotic cells. (K) hsp 70-flp; Ay-Gal4, UAS- $\beta G a l / U A S-N^{D N}$. Experimental conditions are the same as in $H$. Tunel staining (red) is similar to wild type $(J)$. $(L)$ Dorsal view of a wild-type stage 12 embryo. Anterior is to the left. Expression of Lz protein is observed in crystal cell precursors (circled) of the head mesoderm. Additional ectodermal expression of $\mathrm{Lz}$ (arrow) is unrelated to its role in hematopoiesis (Lebestky et al. 2000). Cell counts were performed for 30 bilateral clusters of $\mathrm{Lz}^{+}$cells. $(M)$ Dorsal view of a $N^{55 e 11}$ stage 12 embryo. Fewer Lz expressing crystal cell precursors (circled) are seen. In contrast, Lz expression in the ectoderm is expanded (arrow), due to the neurogenic phenotype of Notch, unrelated to its role in hematopoiesis. In $C, G-H$, and $J-K$, the dotted line marks the outline of the lymph gland lobe. Bars: $A-C, G-H, J-M, 25 \mu \mathrm{m} ; D-F, 10 \mu \mathrm{m}$.

Using the FLP/FRT system (Golic 1991), we generated mutant clones of $N^{55 e 11}$ (Fig. 1D), $N^{t s 1}$ (Fig. 1E), and $\mathrm{Su}(H)^{\Delta 47}$ (Fig. $\left.1 \mathrm{~F}\right)$ in the lymph gland. Clones were marked by the absence of $\beta \mathrm{Gal}$ expression and were always small, reflecting an early requirement for the Notch pathway in cell proliferation. Importantly, $\mathrm{Lz}^{+}$ cells were always excluded from the mutant clones. Approximately $200 \mathrm{Lz}^{+}$cells were counted for each genotype. We also generated positively marked cells within the lymph glands that express an extracellular, dominant negative form of Notch ( $\mathrm{N}^{\mathrm{DN}}$; Rebay et al. 1993). As in the loss-of-function clones, marked $\beta \mathrm{Gal}^{+}$cells expressing $\mathrm{N}^{\mathrm{DN}}$ do not coexpress $\mathrm{Lz}$ (Fig. $\left.1 \mathrm{G}, \mathrm{H}\right)$. Therefore, $\mathrm{Lz}^{+}$ cells are derived preferentially from wild-type precursors, once again suggesting a requirement for Notch in the development of crystal cell precursors. Rare exceptions to this rule have been seen (Fig. 1I), presumably reflecting minor variability in the level of expression of $U A S-N^{D N}$ in individual $\beta \mathrm{Gal}^{+}$cells. Additionally, lymph glands that misexpress $\mathrm{N}^{\mathrm{DN}}$ are smaller than wild-type controls and fewer $\beta \mathrm{Gal}^{+}$cells are seen. This is not a result of increased apoptosis, as the fraction of cells that stain with TUNEL is not increased in $N^{D N}$ lymph glands compared with controls (Fig. 1J,K). Taken together, the above genetic analyses establish that Notch signaling is critical for the specification of the crystal cell lineage, but also has an early role in the proliferation of hematopoietic cells in the lymph gland, similar to the proliferative role of Notch in imaginal discs (Go et al. 1998; Artavanis-Tsakonas et al. 1999).

Notch function is also required for crystal cell devel- opment in the head mesoderm region during embryogenesis. The average number of $\mathrm{Lz}^{+}$crystal cell precursors in each bilateral cluster is reduced significantly in $N^{55 e 11}$ embryos [from 18 in wild-type to 9 in mutant stage 12 embryos $(\mathrm{n}=30)$; Fig. $1 \mathrm{~L}, \mathrm{M}]$. The residual crystal cell development in $N^{55 e 11}$ embryos likely reflects the maternal contribution of Notch (Morel and Schweisguth 2000).

\section{Serrate is the ligand for Notch in hematopoiesis}

To identify the Notch ligand responsible for the development of crystal cells, we investigated the possible role of the two Notch ligands, Delta (DI) and Serrate (Ser) in larval hematopoiesis. Expression of Dl protein was not detected in the lymph gland, and expression of $\mathrm{Lz}$ was not altered in the $D 1^{M 2} / D 1^{R 9}$ genetic background (data not shown). In contrast, $\mathrm{Lz}$ expression was absent in lymph glands of $\mathrm{Ser}^{B d 3} /+$ larvae (Fig. 2A,B), and as a consequence, significantly fewer circulating crystal cells were observed (Fig. 2C,D). As $\operatorname{Ser}^{B d 3}$ is a dominant-negative allele, we used a null allele, $\operatorname{Ser}^{R \times 82}$, to generate loss-of-function clones. Mutant clones usually did not contain Lz-expressing cells (Fig. 2E). However, consistent with Ser being required in signaling cells, rare examples of $\mathrm{Ser}^{-} / \mathrm{Ser}^{-}$mutant cells at the edge of a clone were found to express Lz (Fig. 2F, arrowhead). These results establish Ser as a ligand for Notch in the specification of crystal cells.

To investigate the sufficiency of the Notch pathway in crystal cell development, we used the Ga14/UAS system 


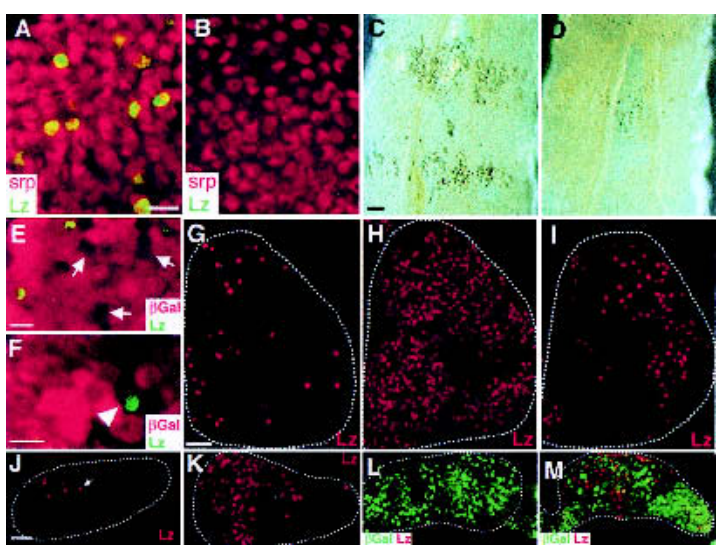

Figure 2. Serrate/Notch signaling is necessary and sufficient for crystal cell development. (A) Wild-type first lymph gland lobe from a third instar larva. Lz protein (green) is expressed in a subset of $\mathrm{Srp}^{+}$ hemocyte precursors (red). (B) $\mathrm{Ser}^{B d 3} /+$. Lz protein is absent, but Srp $($ red) is unaffected. $(C)$ Whole-mount third instar $B c /+$ larva. Crystal cells in circulation and in sessile populations along the dorsal body wall are visible due to precocious melanization in the Black cells $(B c /+)$ genotype. $(D) B c /+$; $S^{B d 3} /+$. Mature crystal cell development is largely suppressed by the dominant Ser mutation. $(E-F)$ hsp 70-flp/ FRT clones of $\operatorname{Ser}^{R \times 82}$. Clones (arrow) are marked by the absence of $\beta \mathrm{Gal}$ (red) expression. $\mathrm{Lz}^{+}$cells (green) are generally excluded from mutant clones $(E)$. However, rare examples $(4$ such cells seen of 92 $\mathrm{Lz}^{+}$cells counted) of $\mathrm{Ser}^{-} / \mathrm{Ser}^{-}$cells at the edge of a mutant clone were found to express $\mathrm{Lz}(F$, arrowhead). $(G-M)$ Ectopic development of crystal cells upon misexpression of $N^{\text {act }}$ or Ser. $(G)$ Wild-type lymph gland stained for Lz protein (red). (H) hsp70-Gal4/UAS-N ${ }^{a c t}$. Transient misexpression of $\mathrm{N}^{\text {act }}$ during the third larval instar causes a dramatic increase in the number of $\mathrm{Lz}^{+}$cells. (I) hsp70-Gal4/+; UAS-Ser/+. Similar misexpression of Ser causes a more moderate increase in $\mathrm{Lz}^{+}$cells. ( $/$) Wild-type second lobe of the third instar lymph gland contains very few $\mathrm{Lz}^{+}$cells (arrow). (K) hsp70-Gal4/ $U A S-N^{a c t}$ second lobe. A large number of $\mathrm{Lz}^{+}$cells are seen upon ectopic activation of the Notch pathway. $(L-M)$ Sustained misexpression of Ser can create ectopic signaling centers. $(L)$ Second lobe from hsp70-flp; Ay-Gal4, UAS- $\beta$ Gal/+ lymph gland is outlined. Random flp-out clones express $\beta \mathrm{Gal}$ (green), but no $\mathrm{Lz}^{+}$cells are seen (red). (M) hsp70-flp; Ay-Gal4, UAS- $\beta$ Gal/+; UAS-Ser/+. In this genotype, $\beta \mathrm{Gal}^{+}$cells (green) also express Ser, causing ectopic development of $\mathrm{Lz}^{+}$cells (red). Bars: $A, B, E, F, 10 \mu \mathrm{m} ; C, D, 100 \mu \mathrm{m} ; G-M$, $25 \mu \mathrm{m}$.

(Brand and Perrimon 1993) to ectopically express an activated form of Notch, N ${ }^{\text {act }}$ (Fortini et al. 1993), or Ser in the lymph glands (Fig. 2G-M). The number of $\mathrm{Lz}^{+}$cells was dramatically increased in hsp70-Gal4/UAS-N ${ }^{a c t}$ lymph glands (Fig. 2G,H). A more modest increase in $\mathrm{Lz}^{+}$ cells was seen when Ser was overexpressed in hsp70Ga14/UAS-Ser larvae (Fig. 2I). Strikingly, misexpression of $\mathrm{N}^{\text {act }}$ caused $\mathrm{Lz}^{+}$cells to appear in the second lobe (Fig. $2 \mathrm{~K})$, where they are rarely seen in wild type (Fig. 2J). To create small clones of cells with sustained misexpression of Ser, we used the AyGal4 system (Ito et al. 1997). In contrast to the $\beta \mathrm{Gal}$ control (Fig. $2 \mathrm{~L}$ ), the misexpression of Ser resulted in a dramatic increase in the number of crystal cell precursors differentiating in the second lobe (Fig. 2M). Thus, the misexpression of Ser can create ectopic signaling centers, which then induce $\mathrm{Lz}$ expression.

\section{Serrate defines a signaling center}

Expression of Ser was monitored using an antibody raised against the Ser protein and using a Ser- $\beta$ Gal re- porter (Bachmann and Knust 1998; Fig. 3). Many Serexpressing cells were found to be clustered at the posterior end. Additionally, scattered Ser ${ }^{+}$cells were also seen throughout the gland (Fig. 3A). A high level of Ser- $\beta$ Gal expression is also observed in the cells at the posterior tip, immediately adjacent to a pericardial cell (Rugendorff et al. 1994; Fig. 3B-D). Given the unique pattern of Ser expression in this discrete cluster of cells, we designate this region as the Posterior Signaling Center (PSC). A number of Ser cells that are adjacent to the PSC appear to have broken off from the cluster (Fig. 3A,F, inset), suggesting that $\mathrm{Ser}^{+}$cells may all be derived from the
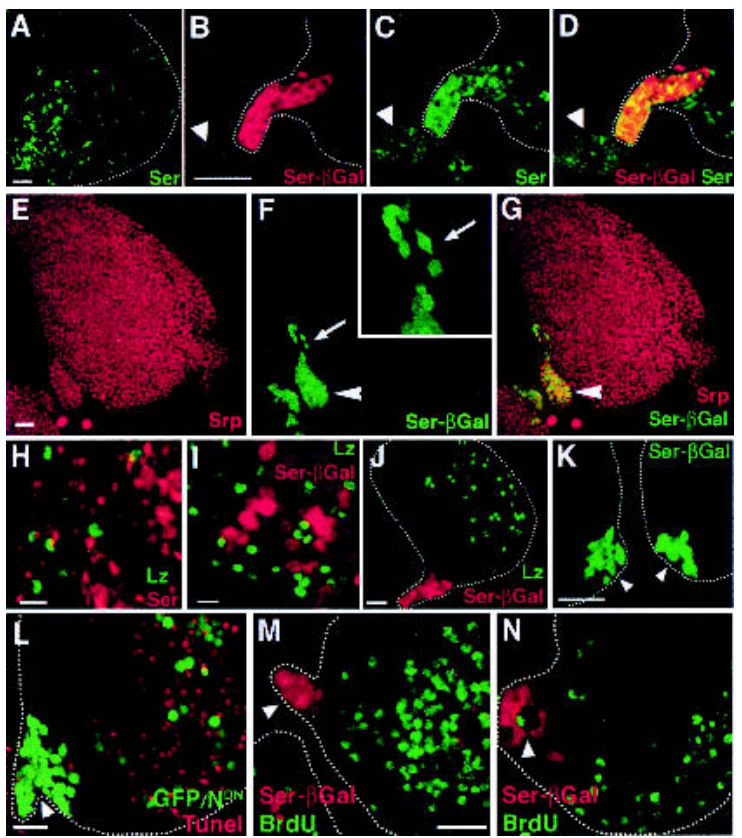

Figure 3. Serrate expression defines a signaling center. $(A)$ Ser protein-expressing cells are seen concentrated near the posterior end of the lymph gland (lower left corner). Additionally, dispersed cells throughout the gland also express Ser protein. $(B-D)$ Identical flattened confocal images of the PSC region of Ser- $\beta$ Gal lymph gland showing Ser- $\beta$ gal expression (red, B), Ser protein expression (green, $C)$, and merged image $(D)$. Ser protein expression and Ser- $\beta$ Gal colocalize in the Posterior Signaling Center (PSC) adjacent to a pericardial cell (arrowhead). (E-G) Identical flattened confocal images of a first lobe, stained for Srp (red, E), Ser- $\beta$ Gal (green, F), and merged $(G)$. Ser- $\beta$ Gal-expressing cells colocalize with Srp in the PSC (arrowhead). $\left(F\right.$, inset) Ser ${ }^{+}$cells that appear to be migrating from the PSC (arrow). $(H-I)$ The expression patterns of $\mathrm{Lz}$ protein (green) and $\mathrm{Ser}^{+}$ cells marked by either Ser protein (red, $H$ ), or Ser- $\beta$ Gal (red, I) are mutually exclusive. Multiple examples of $\mathrm{Lz}^{+}$cells in close proximity to $\beta \mathrm{Gal}^{+}$cells can be seen. Visualization of the lower level of Ser- $\beta$ Gal expression in cells inside the lobe requires increased laser power on the confocal microscope, used here but not in $E-G$. $(J)$ Expression of $\mathrm{Lz}$ (green) in the lymph gland is spatially distinct from the PSC marked by high-level expression of Ser- $\beta$ Gal (red). $(K) N^{t s 1}$; Ser- $\beta$ Gal/+. Ser expression (arrowheads) in the PSC is unaffected in the $N^{t s 1}$ mutant. Two bilaterally symmetric anterior lobes (arrowheads) are shown in this panel. (L) hsp70-flp; Ay-Gal4, UAS-GFP/ $U A S-N^{D N}$. Experimental conditions are the same as in Figure $1 \mathrm{H}$. Cells expressing GFP (green) also express $\mathrm{N}^{\mathrm{DN}}$ and are preferentially observed in the PSC region (arrowhead). Apoptotic cells (red) are not observed in the PSC region. $(M, N)$ Flattened confocal images of Ser$\beta \mathrm{Gal}$ expression (red) marking the PSC and BrdU incorporation (green) in a first lobe of third instar lymph gland. Ser- $\beta$ Gal and BrdU are mutually exclusive in the PSC region (arrowhead). BrdU was incorporated for either $1 \mathrm{~h}$ in vitro $(M)$, or for $18 \mathrm{~h}$ in vivo $(N)$. Bars: $A-G, J-N, 25 \mu \mathrm{m} ; H-I, 10 \mu \mathrm{m}$. 
PSC and actively migrate into the body of the lymph gland to seed differentiation of the crystal cells. Future real-time cell imaging will be necessary to definitely establish cell migration patterns.

Ser $^{+}$cells are seen at the PSC, but many are also found inside the gland. In either case, there is no overlap between cells that express $\mathrm{Lz}$ and Ser (Fig. 3H,I). As Ser function is critical for the development of $\mathrm{Lz}^{+}$cells, these results suggest that cell-cell interaction between the two subpopulations is needed for crystal cell development. Consistent with this notion, many examples of $\mathrm{Lz}^{+}$cells that are immediately adjacent to Ser ${ }^{+}$cells were seen (Fig. 3H,I), suggestive of an inductive relationship between these two subpopulations. When Ser mutant clones were generated in the lymph gland (Fig. 2E,F), we frequently found lymph gland lobes that entirely lack $\mathrm{Lz}^{+}$cells, although the entire gland was not mutant. This further supports the hypothesis that $\mathrm{Ser}^{+}$cells are clustered initially to form a signaling center and later disperse and participate in the induction of $\mathrm{Lz}^{+}$cells.

As with all the other cells in the lymph gland, the PSC cells express Serpent (GATA; Fig. 3E-G). The expression of Ser is the first observed indication that distinguishes the PSC cells from the remainder of the Srp ${ }^{+}$progenitors within the lymph gland. Additionally, the PSC displays a number of interesting characteristics that distinguish this region from the rest of the lymph gland. First, although Ser is required for the expression of Lz, the PSC is spatially distant from the region in which the majority of crystal cells differentiate (Fig. 3J). Second, Ser expression in the PSC is unaffected in $N^{t s 1}$ mutants raised at the nonpermissive temperature (Fig. $3 \mathrm{~K}$ ). Also, in creating clones of $N^{D N}$ in the lymph gland (Fig. $\left.1 \mathrm{H}\right)$, the majority of cells that express $N^{D N}$ are found in the PSC region, and these cells do not apoptose compared with cells in the rest of the lymph gland (Fig. 3L). These results suggest that although the $\mathrm{Ser}^{+}$cells of the PSC signal through the Notch pathway, they themselves are refractory to the Notch signal and do not require Notch for their own development. Finally, the Ser ${ }^{+}$cells of the PSC do not incorporate BrdU administered in vitro for $1 \mathrm{~h}$ (Fig. $3 \mathrm{M})$ or through overnight $(18 \mathrm{~h})$ feeding of third instar larvae (Fig. 3N), unlike the majority of cells within the lymph gland that actively proliferate in the third instar (Fig. 3M,N). Thus, the Ser cells of the PSC represent a distinct signaling cell population that rarely proliferates, but is important for proliferation of hemocyte precursors and for their differentiation into crystal cells.

We next investigated the temporal relationship between Notch signaling and Lz expression. Ser is expressed robustly in the second instar larval lymph gland (Fig. 4A), however, very few $\mathrm{Lz}^{+}$cells, if any, are seen at this stage (Lebestky et al. 2000). Whereas Lz is spatially restricted to the first lobe of the lymph gland, Ser is also seen in further posterior lobes (Fig. 4B). Consistent with these temporal and spatial relationships, expression of Ser is independent of $l z$ (Fig. 4C). The expression of a $12 \mathrm{XSu}(\mathrm{H})-\beta \mathrm{Gal}$ reporter line (Go et al. 1998) can be used as a read-out for Notch signaling. Upon activation of the Notch pathway, the $\mathrm{Su}(\mathrm{H})$ protein causes expression of $\beta \mathrm{Gal}$. In the first lobe of the lymph gland, a small number of cells that receive Notch signal are marked by $\beta \mathrm{Gal}$ expression (Fig. 4D). This expression of $\beta \mathrm{Gal}$ is dependent on Notch (Fig. 4E), but is unaffected in $l z$ null mutants (Fig. 4F). This further substantiates the fact that Notch activation is upstream of $\mathrm{Lz}$ function. When

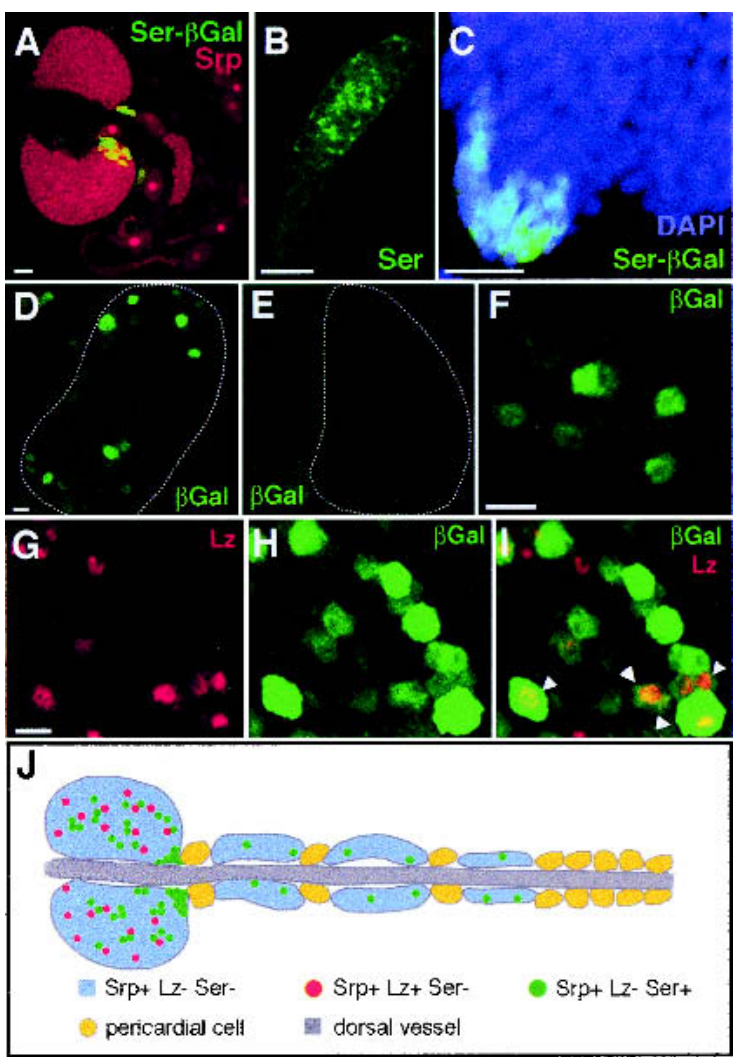

Figure 4. Activation of the Notch pathway precedes Lz expression (A) Ser- $\beta$ Gal (green) and Srp (red) expression in second instar larval lymph glands. $(B)$ Ser protein expression is also observed within the smaller posterior lymph gland of a third instar larva. $\mathrm{Lz}$ is rarely expressed (Fig. 2J). (C) $1 z^{r 15}$; Ser- $\beta G$ Gal $/+$. Ser expression (green) is unaffected in a $l z$ null mutant background. DAPI (blue) marks nuclei within the lymph gland. $(D-E) N^{t s 1} ; 12 X S u(H)-\beta G a l /+$ first lobe from third instar lymph gland. The $12 X S u(H)-\beta G a l$ transgene marks cells receiving the Notch signal (Go et al. 1998). (E) When raised at $29^{\circ} \mathrm{C}$, the nonpermissive temperature, $\beta \mathrm{Gal}$ is no longer expressed. (F) $l z^{r 15} ; 12 X S u(H)-\beta G a l /+. \beta G a l$ expression (green) is maintained in lz null mutant background. $(G-I) 12 X S u(H)-\beta G a l$. Identical confocal images of a third instar lymph gland stained for $\mathrm{Lz}$ protein (red, G), $\beta \mathrm{Gal}$ (green, $H$ ), and merged $(I)$. (I) Arrowheads point to cells that coexpress $\beta \mathrm{Gal}$ and $\mathrm{Lz}$ (see text for details). (J) Schematic representation of third instar larval lymph gland. Four to six pairs of lymph gland lobes along the dorsal vessel (heart) are separated by pericardial cells. Srp is expressed in hemocyte precursors in lymph gland lobes and also in pericardial cells. $\mathrm{Lz}$ is expressed in crystal cell precursors in the first lobe and rarely, if at all, in the further posterior lobes. Ser is expressed at the PSC and also inside the first lobes and other posterior lobes. Bars: $A-E, 25 \mu \mathrm{m} ; F-I, 10 \mu \mathrm{m}$.

lymph glands expressing $12 \mathrm{XSu}(\mathrm{H})-\beta \mathrm{Gal}$ were stained with the $\alpha-\mathrm{Lz}$ antibody (Fig. 4G-I), many examples of $\beta \mathrm{Gal}^{+}$cells that also express Lz protein are seen (Fig. 4I), further supporting that signaling by Notch is important for cells to become $\mathrm{Lz}^{+}$. However, cells that express $\beta \mathrm{Gal}$, but not $\mathrm{Lz}$, and those that express $\mathrm{Lz}$ alone are also seen. The simplest explanation for this pattern is that a cell receiving a Notch signal is a prohemocyte. As a cell initiates $\mathrm{Lz}$ expression, it seems refractory to the Notch signal but the perdurance of the stable $\beta \mathrm{Gal}$ protein is still evident. Finally, as this cell initiates a program for crystal cell differentiation, it continues to express Lz. Mature crystal cells in circulation have been shown to be $\mathrm{Lz}^{+}$(Lebestky et al. 2000). Whether Drosophila hemo- 
cytes undergo maturation as in mammals, can be tested once suitable markers are identified.

Previous studies have shown that a hierarchy of transcription factors is responsible for the differentiation of two independent classes of hemocytes (Lebestky et al. 2000). Results presented here demonstrate that Notch signaling among $\mathrm{Srp}^{+}$hemocyte precursors directly or indirectly regulates the expression of Lz. This signaling creates a molecular distinction between the two major branches of hematopoietic lineage and results in the differentiation of crystal cells (Fig. 4J).

The expression and function of Ser in the lymph gland marks a putative signaling center that highlights certain developmental and molecular similarities between hematopoietic tissues of Drosophila and mammals. The existence of discrete hematopoietic microenvironments within the bone marrow, in which stromal cells influence the proliferation and differentiation of the HSCs has been proposed in many mammalian studies (Bianco et al. 2001). In fact, stromal signaling via the mammalian Ser homolog, Jagged1, has been implicated in the expansion and self-renewal of HSCs (Varnum-Finney et al. 1998). However, in vivo identification and characterization of such signaling microenvironments within the bone marrow is difficult to achieve. Although Drosophila hemocytes are quite distinct from mammalian blood cells, spatial relationships with regards to signaling pathways in the Drosophila lymph gland will lead to a better understanding of how similar patterning events affect HSC populations in the marrow.

In mice, AML1/Runxl is expressed in the embryonic dorsal aorta in hematopoietic clusters associated with an endothelial layer (North et al. 1999). The Notch ligand, Jagged1, is also expressed in the embryonic aorta at this stage (Loomes et al. 1999), but any possible role it may have in the generation of hematopoietic clusters has not been investigated. Furthermore, independent studies have implicated both Notch and AML1 in the progressive commitment and maturation of $\mathrm{T}$ cells (Aster and Pear 2001; Reizis and Leder 2002). The regulatory relationship between Notch and $\mathrm{lz}$ in Drosophila suggests similar interactions may exist between the Notch pathway and AML1 in mammalian hematopoiesis. Investigating such regulatory relationships is especially valuable, as AML1 is critically needed for all definitive hematopoiesis and is also the most frequent target in acute myeloid leukemias (Downing et al. 2000).

\section{Materials and methods}

Flp-out clones expressing $U A S-N^{D N}$ or UAS-Ser were generated using the Ay-Gal4 system (Ito et al. 1997) and marked with either UAS-nuclear $\beta G a l$ or UAS-GFP. To activate $h s p 70-f l p$, progeny were maintained at $18^{\circ} \mathrm{C}$ until the first larval instar, heat-shocked at $37^{\circ} \mathrm{C}$ for $40 \mathrm{~min}$, and then returned to $18^{\circ} \mathrm{C}$ until the dissection. hsp $70-G a 14 / U A S-N^{a c t}$ or hsp 70-Gal4/+UAS-Ser/+ were maintained at $18^{\circ} \mathrm{C}$ until early third instar and heat-shocked twice at $37^{\circ} \mathrm{C}$ for $40 \mathrm{~min}$, then returned to $18^{\circ} \mathrm{C}$ until dissection. $N^{55 e 11}, N^{t s 1}, \mathrm{Su}(H)^{\Delta 47}$, or $\mathrm{Ser}^{R \times 82}$ mutant clones were generated using the hsp70-FLP/FRT system (Golic 1991). Crosses were maintained at $18^{\circ} \mathrm{C}$, heat-shocked for $1 \mathrm{~h}$ in the second instar for $N^{55 e 11}$ and $\mathrm{Su}(H)^{\Delta 47}$, and in the first instar for $N^{t s 1}$ and $\operatorname{Ser}{ }^{R \times 82}$. For Figure 1, $N^{t s 1}$ was raised at $29^{\circ} \mathrm{C}$ either from first instar until third instar, or for $18 \mathrm{~h}$ in the early third instar before dissection in the third instar, in both cases showing loss of $\mathrm{Lz}^{+}$cells.

\section{Acknowledgments}

We thank S. Artavanis-Tsakonas, J. Posakony, F. Schweisguth, Y. Hiromi, K. Irvine, E. Knust, and K. Matthews of the Bloomington Stock
Center for providing fly stocks; R. Reuter and K. Irvine for generously providing antibodies; and Volker Hartenstein and members of the Banerjee laboratory for suggestions and comments. This work was supported by an NIH grant (RO1 HL67395) to U.B. and the U.S. Public Health Service NRSA (GMO7185) to T.L.

The publication costs of this article were defrayed in part by payment of page charges. This article must therefore be hereby marked "advertisement" in accordance with 18 USC section 1734 solely to indicate this fact.

\section{Note added in proof}

Duvic et al. (2002) have also recently studied the role of Ser and Notch in hematopoiesis.

\section{References}

Artavanis-Tsakonas, S., Rand, M.D., and Lake, R.J. 1999. Notch signaling: Cell fate control and signal integration in development. Science 284: $770-776$.

Aster, J.C. and Pear, W.S. 2001. Notch signaling in leukemia. Curr. Opin. Hematol. 8: 237-244.

Bachmann, A. and Knust, E. 1998. Dissection of cis-regulatory elements of the Drosophila gene Serrate. Dev. Genes Evol. 208: 346-351.

Bernardoni, R., Vivancos, B., and Giangrande, A. 1997. glide/gcm is expressed and required in the scavenger cell lineage. Dev. Biol. 191: $118-130$.

Bianco, P., Riminucci, M., Gronthos, S., and Robey, P.G. 2001. Bone marrow stromal stem cells: Nature, biology, and potential applications. Stem Cells 19: 180-192.

Brand, A.H. and Perrimon, N. 1993. Targeted gene expression as a means of altering cell fates and generating dominant phenotypes. Development 118: 401-415.

Daga, A., Karlovich, C.A., Dumstrei, K., and Banerjee, U. 1996. Patterning of cells in the Drosophila eye by Lozenge, which shares homologous domains with AML1. Genes \& Dev. 10: 1194-1205.

Dearolf, C.R. 1998. Fruit fly 'leukemia'. Biochim. Biophys. Acta 1377: M13-M23.

Downing, J.R., Higuchi, M., Lenny, N., and Yeoh, A.E. 2000. Alterations of the AML1 transcription factor in human leukemia. Semin. Cell. Dev. Biol. 11: 347-360.

Duvic, B., Hoffmann, J.A., Meister, M., and Royet, J. 2002. Notch signaling controls lineage specification during Drosophila larval hematopoiesis. Curr. Biol. 12: 1923-1927.

Fortini, M.E., Rebay, I., Caron, L.A., and Artavanis-Tsakonas, S. 1993. An activated Notch receptor blocks cell-fate commitment in the developing Drosophila eye. Nature 365: 555-557.

Fossett, N., Tevosian, S.G., Gajewski, K., Zhang, Q., Orkin, S.H., and Schulz, R.A. 2001. The Friend of GATA proteins U-shaped, FOG-1, and FOG-2 function as negative regulators of blood, heart, and eye development in Drosophila. Proc. Natl. Acad. Sci. 98: 7342-7347.

Go, M.J., Eastman, D.S., and Artavanis-Tsakonas, S. 1998. Cell proliferation control by Notch signaling in Drosophila development. Development 125: 2031-2040.

Golic, K.G. 1991. Site-specific recombination between homologous chromosomes in Drosophila. Science 252: 958-961.

Hoffmann, J.A., Kafatos, F.C., Janeway, C.A., and Ezekowitz, R.A. 1999. Phylogenetic perspectives in innate immunity. Science 284: 13131318.

Ito, K., Awano, W., Suzuki, K., Hiromi, Y., and Yamamoto, D. 1997. The Drosophila mushroom body is a quadruple structure of clonal units each of which contains a virtually identical set of neurones and glial cells. Development 124: 761-771.

Lebestky, T., Chang, T., Hartenstein, V., and Banerjee, U. 2000. Specification of Drosophila hematopoietic lineage by conserved transcription factors. Science 288: 146-149.

Loomes, K.M., Underkoffler, L.A., Morabito, J., Gottlieb, S., Piccoli, D.A., Spinner, N.B., Baldwin, H.S., and Oakey, R.J. 1999. The expression of Jagged1 in the developing mammalian heart correlates with. Hum. Mol. Genet. 8: 2443-2449.

Mathey-Prevot, B. and Perrimon, N. 1998. Mammalian and Drosophila blood: JAK of all trades? Cell 92: 697-700.

Milner, L.A. and Bigas, A. 1999. Notch as a mediator of cell fate deter- 
mination in hematopoiesis: Evidence and speculation. Blood 93: 2431-2448.

Morel, V. and Schweisguth, F. 2000. Repression by suppressor of hairless and activation by Notch are required to define a single row of singleminded expressing cells in the Drosophila embryo. Genes \& Dev. 14: 377-388.

North, T., Gu, T.L., Stacy, T., Wang, Q., Howard, L., Binder, M., Marín Padilla, M., and Speck, N.A. 1999. Cbfa2 is required for the formation of intra-aortic hematopoietic clusters. Development 126: 2563-2575.

Okuda, T., van Deursen, J., Hiebert, S.W., Grosveld, G., and Downing, J.R. 1996. AML1, the target of multiple chromosomal translocations in human leukemia, is essential for normal fetal liver hematopoiesis. Cell 84: 321-330.

Orkin, S.H. 1998. Embryonic stem cells and transgenic mice in the study of hematopoiesis. Int. J. Dev. Biol. 42: 927-934.

Qiu, P., Pan, P.C., and Govind, S. 1998. A role for the Drosophila Toll/ Cactus pathway in larval hematopoiesis. Development 125: 19091920.

Rebay, I., Fehon, R.G., and Artavanis-Tsakonas, S. 1993. Specific truncations of Drosophila Notch define dominant activated and dominant negative forms of the receptor. Cell 74: 319-329.

Rehorn, K.P., Thelen, H., Michelson, A.M., and Reuter, R. 1996. A molecular aspect of hematopoiesis and endoderm development common to vertebrates and Drosophila. Development 122: 4023-4031.

Reizis, B. and Leder, P. 2002. Direct induction of T lymphocyte-specific gene expression by the mammalian Notch signaling pathway. Genes \& Dev. 16: 295-300

Rizki, T.M. and Rizki, R.M. 1981. Alleles of $l z$ as suppressors of the Bc-phene in Drosophila melanogaster. Genetics 97: s90.

Rizki, T.M., Rizki, R.M., and Grell, E.H. 1980. A mutant affecting the crystal cells in Drosophila melanogaster. Roux. Arch. dev. Biol. 188: 91-99.

Rugendorff, A., Younossi-Hartenstein, A., and Hartenstein, V. 1994. Embryonic origin and differentiation of the Drosophila heart. Roux. Arch. Dev. Biol 203: 266-280.

Shrestha, R. and Gateff, E. 1982. Ultrastructure and cytochemistry of the cell types in the larval hematopoietic organ and hemolymph of Drosophila melanogaster. Dev. Growth Differ. 24: 65-82.

Tepass, U., Fessler, L.I., Aziz, A., and Hartenstein, V. 1994. Embryonic origin of hemocytes and their relationship to cell death in Drosophila. Development 120: 1829-1837.

Tsang, A.P., Fujiwara, Y., Hom, D.B., and Orkin, S.H. 1998. Failure of megakaryopoiesis and arrested erythropoiesis in mice lacking the GATA-1 transcriptional cofactor FOG. Genes \& Dev. 12: 1176-1188.

Van Den Berg, D.J., Sharma, A.K., Bruno, E., and Hoffman, R. 1998. Role of members of the Wnt gene family in human hematopoiesis. Blood 92: 3189-3202.

Varnum-Finney, B., Purton, L.E., Yu, M., Brashem-Stein, C., Flowers, D. Staats, S., Moore, K.A., Le Roux, I., Mann, R., Gray, G., et al. 1998 The Notch ligand, Jagged-1, influences the development of primitive hematopoietic precursor cells. Blood 91: 4084-4091.

Wang, Q., Stacy, T., Binder, M., Marin-Padilla, M., Sharpe, A.H., and Speck, N.A. 1996. Disruption of the Cbfa2 gene causes necrosis and hemorrhaging in the central nervous system and blocks definitive hematopoiesis. Proc. Natl. Acad. Sci. 93: 3444-3449.

Weinmaster, G. 2000. Notch signal transduction: A real rip and more. Curr. Opin. Genet. Dev. 10: 363-369.

Werner, M.H., Shigesada, K., and Ito, Y. 1999. Runt domains take the lead in hematopoiesis and osteogenesis. Nat. Med. 5: 1356-1357.

Yoshida, H., Takakura, N., Hirashima, M., Kataoka, H., Tsuchida, K., and Nishikawa, S. 1998. Hematopoietic tissues, as a playground of receptor tyrosine kinases of the PDGF-receptor family. Dev. Comp. Immunol. 22: 321-332. 


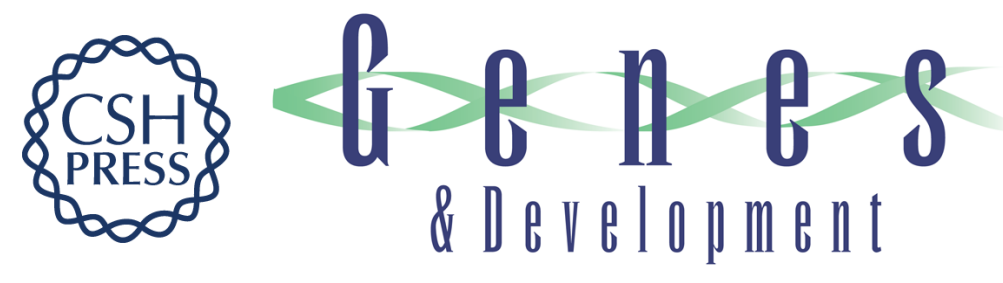

\section{A Serrate-expressing signaling center controls Drosophila hematopoiesis}

Tim Lebestky, Seung-Hye Jung and Utpal Banerjee

Genes Dev. 2003, 17:

Access the most recent version at doi:10.1101/gad.1052803

References This article cites 39 articles, 20 of which can be accessed free at: http://genesdev.cshlp.org/content/17/3/348.full.htmI\#ref-list-1

License

Email Alerting

Receive free email alerts when new articles cite this article - sign up in the box at the top Service right corner of the article or click here.

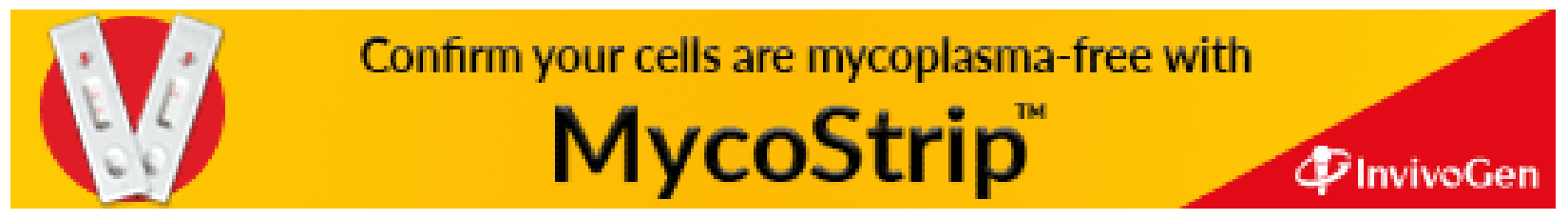

